\title{
STUDYING THE INITIAL STABILITY OF A NEW TYPE OF HIP REPLACEMENT- SPIRON SHORT STEM
}

\author{
Mai Duc Thuan*, Nguyen Quoc Dung*
}

\section{ABSTRACT}

Objective: we perform this study with the following objective: Studying the initial stability of a new type of hip replacement-spiron short stem. Subjects and methods: Experiments to determine the deformability, elasticity and force capacity of the femur were used for the short stem and the long stem replacement corresponds to the original femur without the prosthesis. Along with that, we were evaluated short-term results of treatment for 70 patients with 92 Spiron hips replacement in the Institute of Trauma and Orthopedic-108 Military Central Hospital, from 2012 to 2016. Results: The analysis results showed that the Spiron short stem prosthetic had a significantly smaller displacement in the midfrost bond than the Zweymuller long stem prosthesis. Clinical research: Harris's score was $45.2( \pm 2.4)$ before surgery. At the final examination time Harris's score was $91.4( \pm 2.5)$. The rate: very good $(75.0 \%)$, good $(17.4 \%)$, average $(3.3 \%)$, poor $(4.3 \%)$. There are 02 cases early stem loosening had to stem revision surgery (2.2\%). Conclusion: These results show the good initial fixed ability as well as stability when replaced by the Spiron stem.

Keywords: The initial stability, new hip replacement, spiron short stem.

\section{INTRODUCTION}

Total hip arthroplasty (THA) has become popular in the world and no one can deny its great effectiveness. Recent studies have focused the surgeon's interest on short-stem

*108 Military Central Hospital

Responsible person: Mai Duc Thuan

Email: thuanmd108@gmail.com

Date of receipt: 05/7/2021

Date of scientific judgment: 03/8/2021

Reviewed date: 25/8/2021 replacement designs [1]. 1999, in Germany, Birkenhauer B. created a new type of short stem total hip replacement called the Spiron total hip replacement [2], which was fixed in femoral neck. In general, studies show that the introduction of the spiron short-stem has overcome difficulties and limit complications when revision hip replacement [2][3]. However, there are some doubts about the immobilization ability and clinical outcome of short stem spiron. So that's why we perform this study with the following objective: Studying the initial stability of a new type of hip replacement-Spiron short stem.

\section{SUBJECT AND METHOD}

\subsection{Spiron's Initial Stability Analysis} Method

\subsubsection{Experimental samples}

Experiments were carried out on 48 bones of 24 German shepherd dogs, which were used to measure relative motion. Total bone mineral density (TBMD) was measured before prosthetic implantation to rule out differences in bone density in each pair of femurs. TBMD was measured using filterresorption-based quantitative computed tomography (STRATEC XCT-900) in the calculation of total bone mineral density based on on the linear attenuation factor $\mu$. According to the manufacturer STRATEC the threshold values are $10 / \mathrm{mm}$ and the values range from 0.100 to 0.990 . In this study, cortical bone determined the threshold value of 0.930 and for cancellous bone it was 0.790 [4]. 


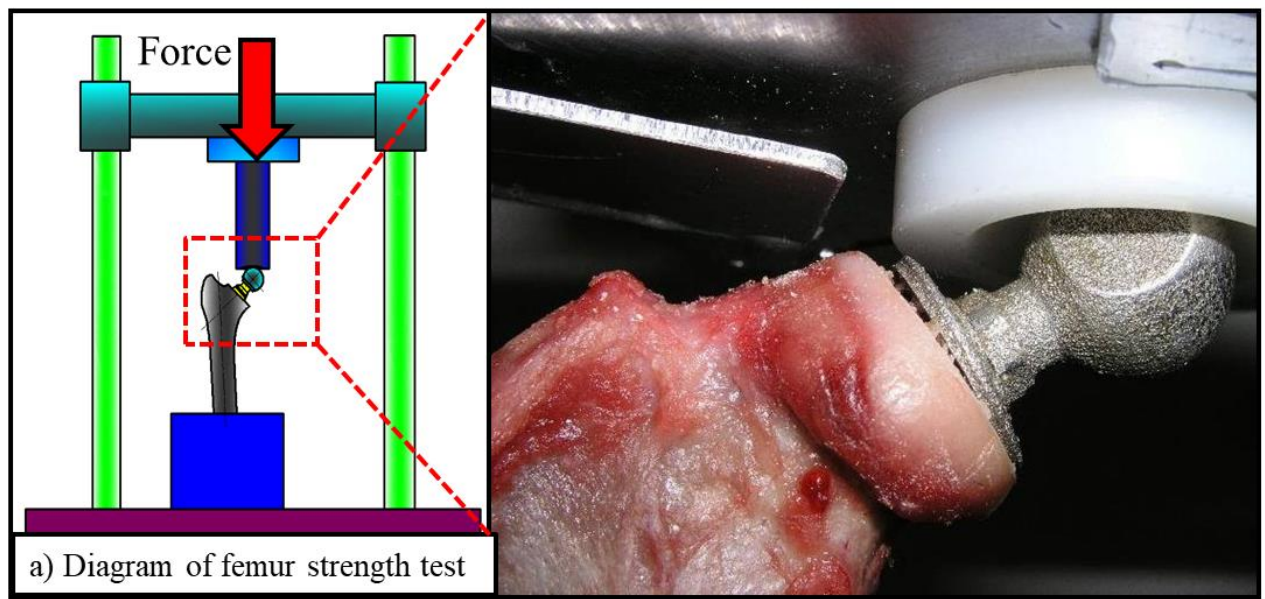

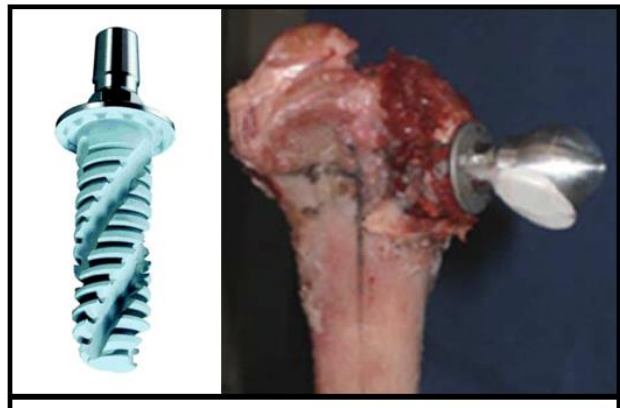

b) Endurance limit with Spiron joint

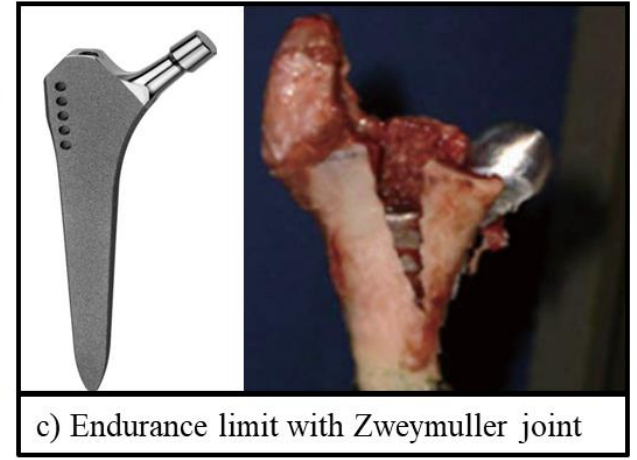

c) Endurance limit with Zweymuller joint

Fig. 1 Diagram of strain determination

The depiction in Fig. 1 shows 48 femurs cut immediately adjacent to their ducts. After implantation of the prosthesis, the remaining end of the femur was immobilized by PMMA and mounted onto a $5 \mathrm{~cm}$ high aluminum cylinder. The femurs are angled $8^{\circ}$ so that they can flex in the anterior plane when subjected to axial loads. In order to compare the displacements between the two types of prostheses, the study was conducted on a pair of bones from the same dog, thereby reducing the influence of anatomical variations.

\subsubsection{Spiron and Zweymuller implants}

The Spiron short stem prosthesis, made of the titanium-vanadium alloy used in the tests, was manufactured by the manufacturer for use in the femur, with no hydroxylapatite precursors. Where the ZweymullerAllosystemic SL long stem prosthesis is a cementless straight-body prosthesis made of Ti6-Al-4V alloy. Zweymuller SL is a prosthetic widely used in the treatment of osteoarthritis in humans. Because of the anchor shell, the Zweymuller SL stem was used in all age groups and femoral shapes [5]. The Zweymuller shaft prosthesis has been miniaturized for experiments with canine femurs. The femoral neck-axis angle of the endothelium was adjusted to accommodate the angular condition of the femur (ie equivalent to an angle of 145$147^{\circ}$ ).

Prosthetics and devices implanted with Spiron prostheses are manufactured to standards and implanted according to the manufacturer's instructions. The locating plate of the drill specimen is placed in the center of the cutting plane and guided into the cancellous bone by means of an implant. 
Using a drill specimen, the central axis is drilled along the axis of the femoral neck. The Spiron prosthetic implant is performed in a clockwise direction. To implant the Zweymuller prosthesis, the complete femoral neck was cut to fit the Zweymuller prosthetic implant model.

\subsubsection{Load and Displacement Analysis}

The displacement between prostheses in the skeletal model was measured by an ultrasound-assisted motion analysis system, CMS 30P-M1008 with data acquisition and processing performed on WinData, used for strain measurement general or movement disorders. The measurement accuracy of the system is $<0.25 \mathrm{~mm}$, with a resolution of $0.085 \mathrm{~mm}$ with the sampling rate is $20 \mathrm{~Hz}$. Only the z-axis values are used because they represent the strain and displacement.

After fixing the femur in the aluminum post with PMMA, it is fixed with screws to the table of the static load tester as shown in Fig 1. The femur is tilted $8^{\circ}$ inwards in the anterior plane to simulate the equivalent load corresponding to the standing position of legs in the normal state [6]. The axial load on the strain gauge is transferred to the femur by the top-down motion of the pressing table. Each measurement is computer-controlled at a speed of $2 \mathrm{~mm} / \mathrm{s}$ and the standard force is controlled at $100 \mathrm{~N} / \mathrm{s}$ and held for a specified time. The parameters obtained after measurement include compressive strength, deformation, displacement, and maximum applied load.

\subsection{Clinical research}

Subject of the study: 70 patients were treated with 92 Spiron hips replacement at the Institute of Trauma and Orthopedic-108 Military Central Hospital from February 2012 to October 2016.
- Selection criteria: Patients who are from 18-50 years old with stage IV, V, VI of avascular necrosis of femoral head (According to Steinberg classification). The shape and quality of femoral neck is good and not deformed, the magnetic resonance imaging (MRI) from the femoral neck has no focal necrosis.

- Exclusion criteria: Remove patients with follow-up time of less than 3 months, no medical records or no follow-up appointment.

Method: Prospective study method, crosssectional description, longitudinal, nocontrols.

Evaluation of result: We examined and assessed hip function based on Harris W.'s scale 100. Evaluation time: Preoperative, 3month postoperative and final examination. The results are classified into four categories: Very good (90-100 points), good (80-89 points), average (70-79 points), poor $(<70$ points). The data was input and processed by program SPSS 15.0, calculated on average ( $\bar{X}$ ), standard deviation (SD), and p value.

\section{RESULTS AND DISCUSSION}

\subsection{Analysis of initial stability when Spiron joint replacement}

The description in Table 1-3 shows that there is a difference between the two groups of joints replaced in terms of behavioral parameters according to strain, displacement and maximum applied load. The magnitude of elastic deformation, displacement with the Spiron prosthesis is higher than with the Zweymuller prosthesis. Especially at $1000 \mathrm{~N}$, the Spiron prosthetic showed significantly increased cyclic behavior compared with the Zweymuller prosthesis $(1.47 \mathrm{~mm}$ and 1.13 $\mathrm{mm})$. The irreversible plastic deformation of 
the Spiron restoration was significantly lower than that of the Zweymuller restoration $(\mathrm{P}=$ 0.05). It represents a significant difference between the groups $(\mathrm{P}<0.05)$. The Spiron prosthesis showed $1.27 \mathrm{~mm}$ deformation at $1000 \mathrm{~N}$, while the Zweymuller prosthesis produced $1.03 \mathrm{~mm}$ corresponding to $400 \mathrm{~N}$ and $3.29 \mathrm{~mm}$ deformation corresponding to $1000 \mathrm{~N}$ such compared to the Spiron and Zweymuller prosthetic is about three times higher. The maximum applied load to fracture was significantly higher for Spiron restorations than for Zweymuller restorations $(\mathrm{P}=0.05)$. Zweymueller and the group without prostheses $\mathrm{N}=32$ ( $\mathrm{P}<0.05)$. The range of maximum applied loads for the two restorations is relatively high, Spiron prosthetics show ranges from 792 to $2200 \mathrm{~N}$ and Zweymuller prostheses from 399 to $1317 \mathrm{~N}$.

The range in the no-prosthesis group shows the largest load-carrying value followed by the Spiron prosthetic and finally the Zweymuller as shown in Figs. $1 b$ and $c$. In which the Spiron prosthesis is minimally destructive, it is tilted far from the bone around the femoral neck and does not affect the underlying bony parts. The Zweymuller prosthesis will show an initial fracture in the proximal part of the femoral shaft, the bone then splits along the length of the bone resulting in mass destruction of the femoral shaft.

Table 1. Average cyclic behavior for different loads by different groups of prostheses

\begin{tabular}{|l|c|c|c|c|c|}
\hline \multirow{2}{*}{ Joint type } & \multicolumn{5}{|c|}{ Cyclic behavior (reversible) } \\
\cline { 2 - 6 } & $\mathbf{2 0 0} \mathbf{~ N}$ & $\mathbf{4 0 0} \mathbf{~ N}$ & $\mathbf{6 0 0} \mathbf{~ N}$ & $\mathbf{8 0 0} \mathbf{~ N}$ & $\mathbf{1 0 0 0} \mathbf{~ N}$ \\
\hline Zweymueller & $0.59(0.37)$ & $0.70(0.40)$ & $0.91(0.54)$ & $1.18(0.57)$ & $1.13(0.86)$ \\
\hline Spiron & $0.56(0.30)$ & $0.75(0.45)$ & $0.96(0.58)$ & $1.22(0.59)$ & $1.47(0.75)$ \\
\hline
\end{tabular}

Table 2. Deformation in different restoration groups

\begin{tabular}{|l|c|c|c|c|c|}
\hline \multirow{2}{*}{ Joint type } & \multicolumn{5}{|c|}{ Subsidence, irreversible (migration) } \\
\cline { 2 - 6 } & $\mathbf{2 0 0} \mathbf{~ N}$ & $\mathbf{4 0 0} \mathbf{~ N}$ & $\mathbf{6 0 0} \mathbf{~ N}$ & $\mathbf{8 0 0} \mathbf{~ N}$ & $\mathbf{1 0 0 0} \mathbf{~ N}$ \\
\hline Zweymueller & $0.49(0.67)$ & $1.03(0.72)$ & $1.78(0.75)$ & $2.58(0.63)$ & $3.29(0.96)$ \\
\hline Spiron & $0.17(0.18)$ & $0.32(0.32)$ & $0.58(0.38)$ & $0.92(0.53)$ & $1.27(0.64)$ \\
\hline
\end{tabular}

Table 3. Maximum force calculated for joint groups

\begin{tabular}{|c|c|c|}
\hline \multirow{2}{*}{ Joint type Parameter } & \multicolumn{2}{|c|}{ Maximal applied load } \\
\hline & Mean & SD \\
\hline Zweymueller & 853.8 & 307.7 \\
\hline Spiron & 1305.5 & 418.4 \\
\hline Non-prothessis & 3935 & 1158.8 \\
\hline
\end{tabular}

This study was conducted to test the initial special design and size. The Zweymuller stability of the Spiron short stem prosthesis compared with the conventional prosthesis. The results of the analysis showed that the difference in the elasticity of the Spiron and Zweymuller prostheses. The lower resilience of Zweymuller restorations is due to the group of restorations showed significantly higher values than Spiron restorations in terms of plastic deformation. This can be explained by the significantly lower elasticity of the Zweymuller prosthetic bone fusion. According to the analysis results, these 
differences are related to the design of the Spiron prosthesis with a conical screw stop, proximal force transfer and small size contribute to the increased dynamic elastomeric motion of the bone when compared to Zweymuller restorations [6]. Zweymuller's dynamic elastic motions are reduced, due to its stiffness, size and shape. Unlike a cylindrical screw, which creates only a stopper around the thread, the conical screw shape of the Spiron prosthesis allows for locking around the thread and the screw flanks. The stress running perpendicular to the axis of the screw causes a more uniform strain distribution with long-term stability [4]. Thus, the rotator fiber of the Spiron prosthesis cuts perpendicularly to the orbit of the femoral neck. The best primary stability is achieved when the contact angle is square between the spongy trabeculae and the implant [6].

\subsection{Surgical results}

The average follow-up time was 34.5 months, the shortest was 3 months, and the longest was 63 months. There are 37 cases in which follow-up time is more than 36 months, accounting for $40.2 \%$.

Table 4. The final examination results classification according to Harris $(\mathrm{n}=92)$

\begin{tabular}{|c|c|c|}
\hline Joints & Number & Proportion (\%) \\
\hline Classification & & 75.0 \\
\hline Very good & 69 & 17.4 \\
\hline Average & 16 & 3.3 \\
\hline Poor & 3 & 4.3 \\
\hline Total & 4 & 100 \\
\hline
\end{tabular}

Table 5. Comparison of Harris score on final examination with postoperative and preoperative $(n=92)$

\begin{tabular}{|c|c|c|c|}
\hline Harris score & $\begin{array}{l}\text { Preoperative (1) } \\
\quad(n=92)\end{array}$ & $\begin{array}{l}\text { Postoperative(2) } \\
(\mathrm{n}=92)\end{array}$ & $\begin{array}{l}\text { Final examination } \\
\quad(3)(n=92)\end{array}$ \\
\hline $\bar{X} \pm \mathrm{SD}$ & $45.2 \pm 2.4$ & $92.1 \pm 3.3$ & $91.4 \pm 2.5$ \\
\hline $\mathrm{p}$ & p1. & $.001 ; \mathrm{p} 1-3<0.001$ & $-3>0.1$ \\
\hline
\end{tabular}

There are 15 cases with light fringe around the stems in the X-ray film on the last examination, accounting for $16.3 \%, 12$ of which were over $2 \mathrm{~mm}$ of displaced stems, accounting for $13.0 \%$. All of the 12 cases are medical angulation of distal fragment.

In Germany, Birkenhauer B. (2003) [1] replaced 38 Spiron hip prosthesis implants for 34 patients, Harris score in the preoperative time was 51 (24-76), after one year follow-up of 20 patients, Harris score was 94 points (86-100). Lugender A. (2012) [3] replaced 28 Spiron hips for 26 patients, Harris score was 55.4 before surgery, after 3 months of testing was 90.5 points.

In Vietnam, from $8 / 2011$ to $5 / 2013$, Nguyen Van Thach (2013) [7] replaced 26 spiron hip joints for 22 patients with excellent and good results (97.2\%). Bui Hai Nam (2015) [8] studied 60 Spiron hip joints, an average of Harris score was $58.78 \pm 8.87$ before surgery. After an average follow-up 
of 21.31 months, Harris scores increased to $89.37 \pm 6.73,98.3 \%$ of patients have good and very good results.

We studied on 92 Spiron hip joints, Harris score was $45.2 \pm 2.4$ before surgery and increased to $92.1 \pm 3.3$ after surgery. At the last examination (average was 34.5 months), Harris score was $91.4( \pm 2.5)$. Specifically, according to table 4 there were $69 / 92$ excellent joints ( $75.0 \%)$, 16/92 good joints (17.4\%), 3/92 medial joints (3.3\%) , 4/92 bad joints $(4,3 \%)$.

Because of the different age of patients studied, kind of diseases, patient caring conditions, it is difficult to accurately compare our results with the authors. But clearly, our research has good result.

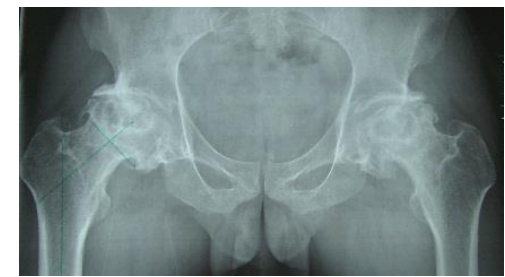

A

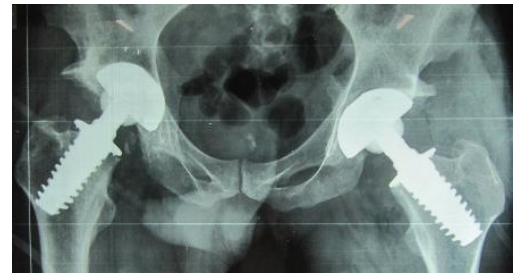

B

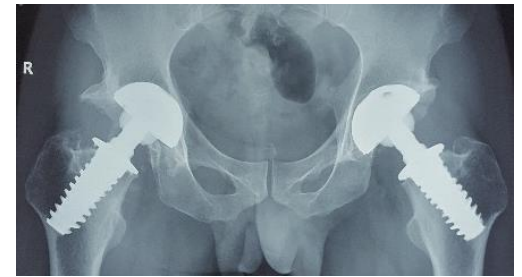

C

Fig.2 Pt Duc VP (A: Preoperative, B: Postoperative, C: Final examination (after 50 months)

There are 15 cases of having light around the joints in the X-ray film at the time of final examination, (16.04\%), among which 12 cases of joint displacement more than $2 \mathrm{~mm}(13.0 \%)$, all of which were angulations. There are 02 cases early stem loosening had to stem revision surgery $(2.2 \%)$. The phenomenon of arthrokatadysis and medical angulation of distal fragment is very noticeable for short stem. In 2013, Banerjee S. [9] reported on the medical angulation of distal fragment rate in general which is $1.4 \%$ $(0-7 \%)$. Based on the outcomes of using Spiron total hip replacement, Stulberg S.D. [10] concluded that the rate of anginal complications was higher; this was a particular concern for short-term hip replacement. However, it has not been shown to affect clinical results. Short stem total hip replacement lacks the extension to the diaphysis. Therefore, it is impossible to facilitate the exact orientation of the stems. In the future, it is necessary to study and create a tool to reduce this relatively high angulate fragment rate. Lastly he wrote: "Short stem total hip replacement with no cement cannot avoid complications, such as: medical angulation of distal fragment, arthrokatadysis, and fragment around stems as all other joints. However, it's essential to study further for the rate and causes of the complication. Finally, follow-up time is needed to assess the fatigue life of the stem."[10]. Hence, our medical angulation of distal fragment rate is higher than other cases and increases with follow-up time. It is needed to monitor closely. Therefore, it is important to observe the medical angulation of distal fragment rate with short stem total hip replacement time.

\section{CONCLUSION}

The Experimental studies analysis results show that the deformation of the Spiron prosthesis is lower along with that of the dynamic elastic deformation and its maximum applied load is higher than that of 
the Zweymuller prosthesis. Spiron prosthetics have better load carrying capacity and stress distribution. To evaluate the real utility of Spiron prosthetics applied to 92 Spiron hip joints of Vietnamese people, an average follow-up time of 34.5 months, we draw that:vHarris's score was $45.2( \pm 2.4)$ before surgery. At the final examination time Harris's score was $91.4( \pm 2.5)$. The rate: very good $(75.0 \%)$, good $(17.4 \%)$, average $(3.3 \%)$, poor $(4.3 \%) .15$ cases $(16.3 \%)$ have bright edges around the joints, in which 12 cases of displacement of the joints more than $2 \mathrm{~mm}$ $(13,0 \%)$, all of which were angulations, so require attention to these cases and this rate in the future. There are 02 cases early stem loosening had to stem revision surgery (2.2\%).

These results show the good initial fixed ability as well as stability when replaced by the Spiron stem. We need to studies on the outcomes of short stems hip replacement with longer follow-up time and more number patients.

\section{REFERENCES}

1. W. H. Kluge, "(vii) Current developments in short stem femoral implants for hip replacement surgery," Orthopaedics and Trauma, vol. 23, pp. 46-51, 2009/02/01/ 2009.

2. B. Birkenhauer, H. Kistmacher, and J. Ries, "Zementfreie

Schenkelhalsschraubenprothese Typ Spiron," Der Orthopäde, vol. 33, pp. 1259-1266, 2004/11/01 2004.

3. A. Lugeder, E. Häring, A. Müller, P. Droste, and J. Zeichen, "[Total hip arthroplasty with the cementless spiron femoral neck prosthesis]," Operative Orthopadie und Traumatologie, vol. 25, pp. 388-397, 2013/08// 2013.

4. U. Wiebking, B. Birkenhauer, C. Krettek, and T. Gösling, "Initial stability of a new uncemented short-stem prosthesis, Spiron (R), in dog bone," Technology and health care: official journal of the European Society for Engineering and Medicine, vol. 19, pp. 27182, 08/15 2011.

5. A. Suckel, F. Geiger, L. Kinzl, N. Wulker, and M. Garbrecht, "Long-term Results for the Uncemented Zweymuller/Alloclassic Hip Endoprosthesis: A 15-Year Minimum FollowUp of 320 Hip Operations," The Journal of Arthroplasty, vol. 24, pp. 846-853, 2009/09/01/ 2009.

6. A. Bistolfi, R. Ferracini, C. Albanese, E. Vernè, and M. Miola, "PMMA-Based Bone Cements and the Problem of Joint Arthroplasty Infections: Status and New Perspectives," Materials (Basel, Switzerland), vol. 12, p. 4002, 2019.

7. Nguyen Van Thach and colleague, Evaluating outcomes of replacement of Spiron hip joints for young patients. Viet Nam Journal of Traumatology and Orthopaedics 2013; (3): page 22-26.

8. Bui Hai Nam, Evaluating outcomes of short stems in total hip replacement with no cement in Viet Duc hospital. Thesis, Hanoi Medical University, 2015: page 70-71.

9. Banerjee S., Pivec R., Issa K. et al., Outcomes of short stems in total hip arthroplasty, Orthopedics, 2013, 36(9), pp. 700-707.

10. Stulberg S. D., Patel R. M., The short stem: promises and pitfalls, The Journal of Bone and Joint Surgery, 2013, 95-B, pp. 57-62. 